\title{
Engineering POLYTAC Nanoparticles for Bioorthogonal Click Reaction-Enforced Protein Degradation and Breast Cancer Therapy
}

Haijun Yu ( $\sim$ hjyu@simm.ac.cn )

Shanghai Institute of Materia Medica, CAS https://orcid.org/0000-0002-3398-0880

jing gao

Qiwen Zhu

Shanghai Institute of Materia Medica, CAS

\section{Bo Hou}

Shanghai Institute of Materia Medica, CAS

\section{Lei Yang}

Nanjing University of Chinese Medicine

\section{Xingyu Jiang}

Nanjing University of Chinese Medicine

\section{Zhifeng Zou}

Shanghai Institute of Materia Medica, CAS

\section{Xutong Li}

Shanghai Institute of Materia Medica, CAS

\section{Zhiai Xu}

East China Normal University

\section{Tianfeng Xu}

Shanghai Institute of Materia Medica, CAS

\section{Mingyue Zheng}

Shanghai Institute of Materia Medica https:// orcid.org/0000-0002-3323-3092

\section{Huixiong Xu}

Tongji University

\section{Article}

Keywords: Polymeric PROTAC, Bioorthogonal reaction, Prodrug nanoparticles, Tumor microenvironment, Combinatory therapy

Posted Date: February 10th, 2022

DOI: https://doi.org/10.21203/rs.3.rs-1197718/v1 
License: (c) (i) This work is licensed under a Creative Commons Attribution 4.0 International License. Read Full License

Version of Record: A version of this preprint was published at Nature Communications on July 26th, 2022. See the published version at https://doi.org/10.1038/s41467-022-32050-4. 


\section{Abstract}

PROteolysis TArgeting Chimeras (PROTACs) have been extensively explored for targeted protein degradation and cancer therapy. However, clinic translation of the conventional small molecular PROTACs is challenged by their unfavorable pharmacokinetic profiles and the systemic toxicity due to ontarget but off-tumor protein degradation. Herein we presented a tumor microenvironment-activatable polymeric PROTAC (namely POLYTAC) nanoplatform for tumor-specific PROTAC delivery and combinatory cancer therapy. The POLYTAC nanoparticles were engineered by integrating metalloproteinase-liable poly(ethylene glycol) shell, intracellular acidity-responsive hydrophobic core and reduction-activatable PROTACs into one nanoplatform. The resultant POLYTAC nanoparticles can specifically accumulate at the tumor site and release the PROTACs inside the tumor cells for protein degradation. The POLYTAC nanoparticles can be further engineered for bioorthogonal click reactionenforced tumor-specific delivery of the PROTACs. In combination with photodynamic therapy, we demonstrated that the bioorthogonal POLYTAC nanoparticles remarkably suppressed tumor growth by synergistically inducing cellular apoptosis of the tumor cells in mouse model of MDA-MB-231 breast cancer. The POLYTAC approach might pave the way for tumor-targeted protein degradation and translation of PROTAC-based cancer therapy.

\section{Introduction}

The heterobifunctional PROteolysis TArgeting Chimeras (PROTACs) hold promising potential for cancer therapy by degrading the onco-proteins, in particular the undruggable target ${ }^{1-3}$. PROTACs are generally composed of a "warhead" binding the protein of interest (POI), a ligand hijacking endogenous E3 ubiquitin ligase, and the linker connecting the warhead and the ligand ${ }^{4-6}$. PROTAC can label the POI with ubiquitin by recognizing the $\mathrm{E} 3$ ligase and subsequently, degrade the POI with the ubiquitin-proteasome system (UPS) ${ }^{7-9}$. Compared to the small molecular inhibitors, PROTAC can potentially degrade any intracellular proteins, including those undruggable targets (e.g., transcriptional factors and scaffold proteins) ${ }^{10-12}$. Furthermore, PROTACs are potent for circumventing acquired drug resistance via degrading whole proteins with low drug exposure time and dosage ${ }^{13-14}$. Despite promising, the conventional small molecular PROTACs generally display unfavorable pharmacokinetics and lack of tumor specificity, which might cause systemic toxicity due to their non-specific distribution in the normal tissues $^{15-16}$. It remains a formidable challenge to achieve tumor-specific delivery and potentiate the antitumor potency of the conventional PROTACs.

To achieve tumor-targeted delivery of the PROTACs, several ligand-modification strategies (e.g., antibodyPROTACs, folate-PROTAC and Aptamer-PROTAC conjugates) have been investigated in past years ${ }^{17-22}$. With the assistance of these ligand "gunsight", the decorated PROTACs showed increased cellular uptake in the tumor cells in vitro. In particular, the Aptamer-PROTAC conjugates displayed increased tumor accumulation and antitumor potency than the conventional PROTACs. Nevertheless, these ligandPROTAC conjugates suffer from low serum stability, limited tumor penetration and heterogeneous 
expression of the receptors in different tumor cells and cancer types. Furthermore, opto-PROTACs were developed for ultraviolet light-inducible protein degradation ${ }^{23-24}$. These photo-activatable PROTACs were demonstrated for spatiotemporally controllable protein degradation in vitro. However, clinical translation of the opto-PROTACs was restricted by the poor tissue penetration profile of the ultraviolet light. Therefore, precise PROTACs delivery to the tumor and efficient POI degradation inside the tumor cells remains a formidable challenge.

To this end, we proposed a rationally-designed polymeric PROTAC (POLY-PROTAC) nanotherapeutic for tumor-targeted delivery of the conventional PROTACs and degradation of bromodomain and extraterminal (BET) protein BRD4. The POLY-PROTAC nanoparticles (NPs) were engineered by utilizing the extracellular and intracellular signals (e.g., acidity, enzyme, and reduction) in the tumor microenvironment ${ }^{25-27}$. We first synthesized four VHL-based RPOTACs for BRD4 degradation, and then prepared a series of POLY-PROTACs by reversible addition-fragmentation chain transfer (RAFT) copolymerization approach. The amphiphilic POLY-PROTACs were self-assembled into micellar NPs for systemic delivery of the BRD4 PROTACs (Fig. 1A). To enforce tumor specificity of the POLY-PROTAC, an extracellular tumor acidity (i.e., $\mathrm{pH}=6.5 \sim 6.8$ )-activatable pretargeted NP was subsequently engineered for tumor-specific delivery of dibenzocyclooctyne (DBCO) groups. The azide-modified POLY-PROTAC NPs were thus trapped inside the extracellular matrix of the tumor via in-situ click reaction between the DBCO and azide derivatives. The POLY-PROTAC NPs can be relieved in the tumor mass via extracellular matrix metalloproteinase-2 (MMP-2)-mediated cleavage of the PEG corona. Upon internalization into the tumor cells, the POLY-PROTAC NPs were dissociated inside the acidic endocytic vesicles (i.e., $\mathrm{pH}=5.5 \sim 6.5$ ), and released the PROTAC payload via GSH-mediated reduction of the disulfide bond (Fig. 1B). We further demonstrated that the clickable POLY-PROTAC NPs synergistically induced apoptosis of the tumor cells in combination with photodynamic therapy (PDT) in a mouse model of MDA-MB-231 breast cancer (Fig. 1C). This study might provide a generalizable POLY-PROTAC nanoplatform for tumor-specific PROTAC delivery and potentiated cancer therapy.

\section{Synthesis And Characterization Of The Poly-protac Nps}

The BET family proteins, in particular BRD4, have been investigated as promising antitumor targets due to their crucial role for gene transcription ${ }^{28}$. To design the BRD4-targeted POLY-PROTAC, we selected von Hipel-Lindau (VHL) ligand for PROTAC synthesis since the hydroxyl group in VHL can be reversibly caged via a disulfide spacer. The VHL protein binding ability of the modified PROTACs can be restored by GSHtriggered cleavage of the disulfide bond in the cytosol of tumor cells.

We first synthesized four VHL-based PROTACs by adjusting chemical structures of the VHL ligand and linkers (Fig. 2A, Supplementary Scheme 1-4 and Figs. 1-11). Western blot assay validated that all the four PROTACs remarkably degraded BRD4 in MDA-MB-231 breast cancer cells in vitro, and consequently suppressed downstream c-Myc expression. Noticeably, compounds ARV771 and MZ1 more efficiently reduced BRD4 expression compared to their analogs. The half-degradation concentration $\left(D_{50}\right)$ of ARV771 was 1.9-fold lower than that of mARV771 (Figs. 2B, C and Supplementary Fig. 12). CCK-8 assay 
displayed that all four PROTACs comparably inhibited proliferation of MDA-MB-231 cells. Of note, the half inhibition concentration $\left(\mathrm{IC}_{50}\right)$ of the PROTACs were tens of times lower the BRD4 inhibitor JQ1 (Fig. 2D), suggesting BRD4 degrader more significantly impaired proliferation of the tumor cells than BRD4 inhibitor.

Molecular docking revealed that the hydroxyl in the VHL ligand form H-bond with HIE-115 and SER-111 in the binding pocket of VHL protein (Fig. 2E), verifying the crucial role of hydroxyl group for VHL protein binding. The hydroxyl group of ARV771 and MZ1 was thus methacrylated with a disulfide spacer (termed as Me-ARV771 and Me-MZ1) (Fig. 2F) to reversibly disrupts the interaction and abolish the protein degradation performance of the PROTAC. A reduction-inert ethylene glycol spacer was employed to synthesize the GSH-non-responsive control (namely Me-O-ARV771) (Supplementary Scheme 5, 6). Successful synthesis of the methacrylate-modified PROTACS was validated by nuclear magnetic resonance ( $\left.{ }^{1} \mathrm{H}-\mathrm{NMR}\right)$ and mass spectrometry (MS) measurements, respectively (Supplementary Figs. 1317). High performance liquid chromatography (HPLC) examination displayed ARV771 was completely restored from Me-ARV771 upon $6.0 \mathrm{~h}$ incubation with dithiothreitol (DTT). In contrast, Me-O-ARV771 bearing ethylene glycol spacer kept stable throughout the experimental period (Fig. $2 \mathrm{G}$ and Supplementary Fig. 18), verifying superior reduction sensitivity of the disulfide bond-incorporated MeARV771.

With the reduction-activatable Me-ARV771 and Me-MZ1 in hand, we next sought to synthesize the POLYPROTAC via PEG-induced RAFT copolymerization of Me-ARV771 (or Me-MZ1) and ionizable DPA monomer for intracellular acidity and reduction-triggered delivery of the BRD4 PROTACs. The resultant POLY-PROTACs were termed as PD7 (based on Me-ARV771) or PDM (based on Me-MZ1) according to the PROTAC molecular used. An MMP-2-cleavable GPLGLAG (GG) heptapeptide spacer was introduced into the PD7 POLY-PROTAC (namely PGD7) for prompting tumor-specific accumulation and cellular uptake of the POLY-PROTAC NPs (Supplementary Scheme 7, 8 and Figs. 19-24). Two MZ1-based POLY-PROTACs without GG peptide (PDM), and GG peptide spacer (PGDM) were also synthesized for comparison (Supplementary Scheme 9 and Figs. 25, 26). A reduction-insensitive POLY-PROTAC was also synthesized with Me-O-ARV771 (termed as PGD07, Supplementary Scheme 12 and Fig. 31). All the synthesized POLYPROTACs displayed well-controllable polymerization degrees and narrow molecular weight distribution as determined by ${ }^{1} \mathrm{H}-\mathrm{NMR}$ and gel permeability chromatography measurements, respectively (Supplementary Table 1).

The POLY-PROTAC micellar NPs were then prepared via nanoprecipitation method as described previously ${ }^{29-30}$. At neutral $\mathrm{pH}$ of 7.4 , dynamic light scattering (DLS) data and transmission electron microscopy (TEM) examinations revealed averaged hydrodynamic diameter of $\sim 55 \mathrm{~nm}$ and spherical morphology of the POLY-PROTAC NPs with narrow particle size distribution (polydispersity index (PDI) < 0.2). In contrast, amorphous aggregates appeared at $\mathrm{pH}$ of 6.0 due to acid-induced protonation of DPA groups and dissociation of the POLY-PROTAC NPs (Figs. 2H, I). 
To demonstrate acid-responsive property of the POLY-PROTAC NPs, we then synthesized Pheophorbide A (PPa, a fluorescence dye and well-studied photosensitizer)-labeled $\mathrm{mPEG}_{113}-\mathrm{GG}-b-\mathrm{P}\left(\mathrm{DPA}_{50}-\mathrm{r}-\mathrm{HEMA}_{5}\right)$ (termed as PGDA) diblock copolymer via RAFT copolymerization (Supplementary Scheme 10, 11 and Figs. 27-30). The ARV771 or MZ1-conjugated POLY-PROTAC was then co-assembled with PGDA to obtain fluorophore-labeled POLY-PROTAC NPs. At neutral pH 7.4, the PPa-labeled POLY-PROTAC NPs displayed quenched fluorescence emission due to homo-fluorescence resonance energy transfer between $\mathrm{PPa}$ molecules ${ }^{31}$. On the contrary, the fluorescence emission was remarkably recovered at acidic $\mathrm{pH}$ of 6.2 , mimicking the acidic microenvironment of the endosome organelles ( $\mathrm{pH}=5.8 \sim 6.5)$ (Fig. 2J). This phenomenon further validated acid-triggered dissociation of the POLY-PROTAC NPs, which might facilitate PROTAC release inside the tumor cells.

HPLC examination displayed that in the absence of GSH, ARV771 was marginally released from the PGD7 NPs at either neutral or acidic condition (i.e., pH $=7.4$ or 6.0) (Fig. 2K). In contrast, ARV771 was remarkably restored with the addition of $10 \mathrm{mM} \mathrm{GSH}$. For instance, over $50 \%$ of ARV771 was released upon $4 \mathrm{~h}$ incubation with $10 \mathrm{mM}$ of GSH solution at $\mathrm{pH}$ of 6.0 , which was $\sim 20 \%$ higher than that determined at neutral $\mathrm{pH}$. This phenomenon could be explained by the increased GSH accessibility when the POLY-PROTAC NPs was dissociated at acidic condition.

\section{BRD4 degradation with the POLY-PROTAC NPs in vitro}

To demonstrate the advantage of the MMP-2-sheddable POLY-PROTAC for increased cellular uptake and deep tumor penetration (Fig. 3A), the PGDA7 (with GG peptide spacer) and PDA7 (without GG peptide spacer) NPs were pre-treated with MMP-2 for $1 \mathrm{~h}$ to cleave the PEG corona as reported previously ${ }^{32}$. MDAMB-231 breast tumor cells were then incubated with PDA7 or the dePEGylated PGDA7 NPs for the desired time durations. Flow cytometry measurement identified $\sim 2.8$-fold higher intracellular fluorescence signal of the PGDA7 group than the PDA7 one (Fig. 3B). Confocal laser scanning microscopy (CLSM) examination further revealed remarkably higher intracellular uptake of the PGDA7 NPs than the PDA7 control when examined at $12 \mathrm{~h}$ incubation (Fig. $3 \mathrm{C}$ and Supplementary Fig. 32), validating that MMP-2triggred dePEGylation prompted internalization of the POLY-PROTAC NPS.

The tumor penetration profile of the PPa-labeled POLY-PROTAC NPs was then investigated in threedimensional (3D) multicellular spheroid (MCS) model of the MDA-MB-231 tumor in vitro. CLSM examination showed that upon $12 \mathrm{~h}$ incubation, the MMP-2-insensitive PDA7 NPs were primarily entrapped in the peripheral area, and marginally penetrated into deep area of the MCSs (Figs. 3D, E). In contrast, the sheddable PGDA7 NPs highly diffused into the central area of the MCSs with 3.0-fold higher fluorescence intensity at depth of $50 \mu \mathrm{m}$ (Fig. 3F), implying remarkable tumor penetration profile of the sheddable POLY-PROTAC NPS.

With the MMP-2-liable POLY-PROTAC NPs in hand, we next evaluated their BRD4 degradation performance in vitro. Upon $24 \mathrm{~h}$ incubation, the GSH-activatable POLY-PROTACs of ARV771 and MZ1 efficiently reduced BRD4 protein in MDA-MB-231 tumor cells in vitro (Figs. 3G-J). In contrast, the PGD07 
POLY-PROTAC bearing ethylene glycol linker negligibly affected BRD4 expression (Fig. 3K), validating the crucial role of GSH-triggered reduction of the disulfide bond and restoration of the VHL ligand for protein degradation inside the tumor cells. MMP-2 pretreatment of the MMP-2-sheddable POLY-PROTAC NPS remarkably reduced the $\mathrm{DC}_{50}$ of the PGD7 and PGDM NPs, which were 1.9- and 3.1-fold lower the MMP-2insensitive PD7 and PDM counterparts respectively, and comparable to that of the free ARV771 and MZ1 (Fig. 3L). This could be attributed to increased cellular uptake of the PGD7 and PGDM NPs upon MMP-2mediated cleavage of the PEG corona (Supplementary Fig. 33).

Co-treatment with proteasome inhibitor MG132 abolished the protein degradation ability of the small molecular PROTACs (e.g., MZ1 and ARV771) and the POLY-PROTAC NPs (e.g., PGDM and PGD7) (Fig. 3M), verifying ubiquitin-proteasome-dependent BRD4 degradation profile of the POLY-PROTAC NPs. CCK-8 assay further revealed increased cytotoxicity of the PGDM and PGD7 NPs than that of the MMP-2 non-responsive PGD07 control upon $72 \mathrm{~h}$ incubation (Fig. 3N). Collectively, above data demonstrated that the POLY-PROTAC NPs with sheddable PEG corona and reduction-liable linker efficiently degraded the POI and suppressed tumor cell proliferation in vitro.

\section{Biodistribution and antitumor performance of the POLY-PROTAC NP in vivo}

The tumor-specific accumulation and penetration of the POLY-PROTAC NPs was next investigated in MDA-MB-231 breast tumor-bearing BALB/c nude mouse model in vivo. The MMP-2-sheddable PGDA7 and their MMP-2-insensitive PDA7 analog NPs were administrated via intravenous (i.v.) injection at an identical ARV771 dose of $10 \mathrm{mg} / \mathrm{kg}$ and PPa dose of $5.0 \mathrm{mg} / \mathrm{kg}$ respectively when the tumor volume reached $200 \mathrm{~mm}^{3}$. Fluorescence imaging in vivo displayed that both the PGDA7 and PDA7 NPs accumulated at the tumor site over time via passive tumor-targeting property of NPs (Fig. 4A). Noticeably, the PGDA7 NPs showed significantly higher intratumoral fluorescence intensity and slower decline than PDA7 at all the time points. For instance, the PGDA7 group was of 2.0-fold higher tumor fluorescence intensity than the PDA7 control at $36 \mathrm{~h}$ post-injection (Fig. 4B).

Tumor-specific distribution of the PGDA7 NPs was confirmed by fluorescence imaging of the major organs (e.g., heart, liver, spleen, lung and kidney) and the tumor tissue ex-vivo at $48 \mathrm{~h}$ post-injection (Fig. 4C). CLSM examination of the tumor section further illustrated that the PDA7 NPs distributed in the peripheral area of the blood vessels. In contrast, the PGDA7 NP diffused throughout the tumor tissue (Fig. 4D). Taken together, the fluorescence imaging and CLSM examination data verified increased tumor accumulation and penetration of the PGDA7 NPs due to MMP-2-mediated cleavage of the PEG corona.

We subsequently explored the anti-tumor efficacy of the POLY-PROTAC NPs in MDA-MB-231 tumor model in vivo. The tumor-bearing $\mathrm{BLAB} / \mathrm{c}$ nude mice were randomly grouped when the tumor volume reached $100 \mathrm{~mm}^{3}(\mathrm{n}=5)$, and i.v. injected with PBS, ARV771 or the PGD7 NP at an identified ARV771 dose of 10 $\mathrm{mg} / \mathrm{kg}$. The treatments were repeated every three days for five times (Fig. 4E). Free ARV771 marginally suppressed MDA-MB-231 tumor growth. In contrast, PGD7 NP significantly delayed $~ 50 \%$ of tumor growth and consequently elongated the survival of the tumor-bearing mice (Figs. 4F, G). 
Immunohistochemistry $(\mathrm{IHC})$ examination of the tumor section displayed that free ARV771 marginally affected BRD4 expression (Fig. 4I). In contrast, PGD7 NP remarkably suppressed BRD4 expression with 4-fold lower BRD4-positive area than free ARV771 (Fig. 4J).

To elucidate the mechanism underlying the antitumor performance of the POLY-PROTAC NPS, BRD4 degradation in the tumor lysates was evaluated by western blot assay. Fig. 4K validated that the PGD7 NPs dramatically degraded BRD4 protein (Supplementary Fig. 34). Furthermore, PGD7 NP-treatment significantly elicited the expression of cleaved-caspase-3 both in vitro and in vivo (Fig. 4L), verifying BRD4 degradation induced apoptosis of the tumor cells since caspase-3 is a crucial executor of apoptosis $^{33}$. BRD4 degradation-induced apoptosis of the tumor cells was further verified by Hematoxylineosin (H\&E) staining of the tumor sections ex-vivo (Fig. 4H). There is no body weight loss was found during the whole experimental periods (Supplementary Fig. 35), and hematoxylin-eosin (H\&E) staining of the major organs revealed negligible histopathological damage of the major organs (Supplementary Fig. 35), suggesting good biosafety of the POLY-PROTAC NPs.

\section{Bioorthogonal click reaction amplified tumor distribution of the POLY-PROTAC NPs in vivo.}

Insufficient tumor distribution of the PROTAC is one of the bottlenecks for PROTAC-based cancer therapy ${ }^{2}$. To further prompt tumor-specific accumulation of the POLY-PROTAC NPs (Fig. 5A), we next designed an extracellular tumor acidity-activatable pretargeted NP for tumor-specific delivery of the dibenzocyclooctyne (DBCO) groups. In parallel, azide groups were modified on the surface of the POLYPROTAC NPs for in-situ bioorthogonal click reaction-amplified tumor accumulation and retention of the POLY-PROTAC NPs (Fig. 5A, see Supplementary Scheme 14 and Fig. 39 for synthesis of the $\mathrm{N}_{3}-\mathrm{PEG}_{113^{-}}$

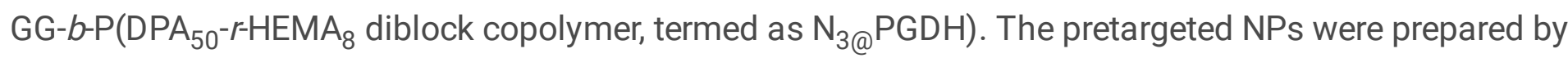
self-assembly of DBCO-modified mPEG-b-poly(ethylene propyl amine) (namely PED) diblock copolymer (Supplementary Scheme 13 and Figs. 36, 37 for diblock copolymer synthesis).

The PED pretargeted NPs displayed homogeneous and spherical morphology with an averaged hydrodynamic diameter of $\sim 60 \mathrm{~nm}$ at the neutral $\mathrm{pH}$ value (i.e., 7.4) (Fig. 5B), and dissociated dramatically at acidic $\mathrm{pH}$ (i.e., 6.5) mimicking the acidic tumor microenvironment (Fig. 5C). The PPaconjugated PED NPs displayed quenched fluorescence at neutral condition (Supplementary Scheme 13 and Fig. 38). In contrast, the fluorescence of PPa recovered at $\mathrm{pH}$ value lower than 6.6 via extracellular acidity-induced dissociation of the pretargeted NPs (Fig. 5D), validating superior tumor extracellular acidity-responsive property of the pretargeted NPs.

To demonstrate extracellular acidity-triggered bioorthogonal click reaction, the DBCO-loaded PED pretargeted NP and the azide-modified $\mathrm{N}_{3} @ P$ PD7 POLY-PROTAC NPs were incubated together at pH of 7.4 or 6.5 , respectively. DLS examination showed uniform hydrodynamic diameter and narrow particle size distribution of the mixed pretargeted and POLY-PROTAC NPs in the FBS-containing neutral buffer solution ( $\mathrm{pH}$ 7.4), suggesting negligible interaction between the PED and the $\mathrm{N}_{3} @ P G D 7$ NPs since the DBCO groups were encapsulated inside the hydrophobic core of the PED NPs (Supplementary Fig. 40). In 
contrast, DLS and TEM examination revealed the appearance of amorphous aggregates with broad size distribution at acidic $\mathrm{pH}$ of 6.5 (Figs. 5E, F). This phenomenon validated the bioorthogonal click reaction between the DBCO and azide groups to form cross-linked nanostructure between the PED copolymer and azide-functionalized POLY-PROTAC NPs.

We next sought to investigate whether bioorthogonal click reaction can increase intratumoral accumulation and retention of the POLY-PROTAC NPs in vivo. Fluorescence imaging in vivo displayed obvious fluorescence signal at the tumor site when the MDA-MB-231 tumor-bearing mice were i.v. injected with the PED pretargeted NPs (Fig. 5G), which was caused by tumor acidity-triggered dissociation of the PED NPs and activation of the PPa fluorescence signal via protonation of the EPA tertiary amine (Fig. 5G). CLSM examination of the tumor sections showed that the PED pretargeted NPs colocalized well with the cell membrane (labeled with wheat germ agglutinin, WGA) 2 4h post-injection, verifying that the PED NPs dissociated and exposed the DBCO groups in the extracellular matrix (ECM) of the tumor tissue (Fig. $5 \mathrm{H}$ ). which could facilitate click reaction between the DBCO and azide groups presenting on the surface of the POLY-PROTAC NPs in the ECM.

To evaluate whether the in-situ click reaction enhance tumor accumulation of the PROTAC molecules in vivo, the $\mathrm{N}_{3} @ P G D 7$ POLY-PROTAC NPs was i.v. administrated $2 \mathrm{~h}$ post-injection of the pretargeted NPs. Fluorescence imaging in vivo displayed much brighter fluorescence signal at the tumor site of the PED + $\mathrm{N}_{3} @ P G D A 7$ group compared to the $\mathrm{N}_{3} @ P G D A 7$-injected mouse group (Fig. 5I). For instance, PED + $\mathrm{N}_{3} @ P G D A 7$ group showed 2.0-fold higher fluorescence signal than the $\mathrm{N}_{3} @ P G D A 7$ NP alone at 24-36h post-injection (Fig. 5K).

Increased tumor distribution of the POLY-PROTAC NPs was validated by fluorescence imaging of the major organs and tumor tissues ex-vivo $48 \mathrm{~h}$ post-injection (Fig. 5L). CLSM examination of the tumor sections demonstrated increased tumor accumulation and deep penetration of the $\mathrm{N}_{3} @$ PGDA7 POLY PROTAC NPs when administrated post i.v. injection of the pretargeted PED NPs (Fig. 5J). HPLC examination of ARV771 distribution in-vivo further demonstrated increased intratumoral distribution and retention of the POLY-PROTAC NPs via in-situ click reaction. In comparison with free ARV771 and $\mathrm{N}_{3} @ P G D A 7$ NPs, the combination of PED + $\mathrm{N}_{3} @ P G D A 7$ NP remarkably enhanced tumor distribution of ARV771 by 3.9- and 1.9-fold respectively, when examined at $36 \mathrm{~h}$ post-injection (Fig. 5M). Both fluorescence imaging and HPLC data provided consistent evidence that the in-situ click reaction significantly enhanced tumor accumulation and retention of ARV771 PROTAC at the tumor site.

\section{Bioorthogonal POLY-PROTAC NPs regressed breast tumor growth in vivo}

Given significantly improved tumor distribution of the clickable POLY-PROTAC NPs, we next investigated their antitumor performance in MDA-MB-231 tumor model in vivo (Fig. 6A). Compared to free ARV771 or $\mathrm{N}_{3} @ P G D 7$ NPs alone, the combination of the PED pretargeted and $\mathrm{N}_{3} @ P G D 7$ NPs much more efficiently suppressed $\sim 70 \%$ of MDA-MB-231 tumor growth (Fig. 6B), and elongated the survival of the tumorbearing mice (Fig. 6C), which can be attributed to increased ARV771 distribution in the tumor tissue. 
In previous studies, we had explored intracellular acidity-activatable PDT for circumventing multidrug resistance of the breast tumor ${ }^{34}$. To explore the potential of the POLY-PROTAC NPs as a generalizable nanoplatform for combinatory therapy, we next sought to combine the bioorthogonal NPs for PDTenforced antitumor therapy (Fig. 6D). The $\mathrm{N}_{3} @ P G D A 7$ NPs integrating the ARV771 POLY-PROTAC and PPa was prepared by co-assembling $\mathrm{N}_{3} @ P G D H$, PGD7 and PGDA diblock copolymers. Western blot assay displayed that combination of BRD4 degradation and PDT in vitro significantly activated the caspase-3 protein in vitro, implying cumulative antitumor performance of the $\mathrm{N}_{3} @ P G D A 7$ NPs (Fig.6E and Supplementary Figs. 41, 42).

The anti-tumor performance of combinatory PDT and BRD4 degradation was next investigated in vivo. The MDA-MB-231 tumor-bearing BALB/c nude mice were randomly grouped when the tumor volume reached $\sim 100 \mathrm{~mm}^{3}$ and treated with PBS, ARV771, PED + N3@ @PGDA + Laser, PED + N 3 @PGD7, PED + PGDA7 + Laser, or PED + N ${ }_{3} @ P G D A 7$ + Laser, respectively (Fig.6F). The $\mathrm{N}_{3} @ P G D A 7$ NP was i.v. administrated $2 \mathrm{~h}$ post-injection of the PED NP, and $671 \mathrm{~nm}$ laser irradiation was applied $36 \mathrm{~h}$ postinjection of the $\mathrm{N}_{3} @ P G D A 7$ NP. Fig. 6G demonstrated that PDT or ARV771 alone marginally suppressed proliferation of the MDA-MB-231 tumor. In contrast, the combination of the bioorthogonal NPs (PED + $\mathrm{N}_{3} @ P G D A 7$ ) and PDT dramatically regressed $90 \%$ of tumor growth, 1.5-fold more efficient than BRD4 degradation alone by PED + $\mathrm{N}_{3} @ P G D A 7$.

Furthermore, combination of the PED pretargeted NPs with $\mathrm{N}_{3} @ P G D A 7$ and PDT elongated the survival of the tumor-bearing mice by $40 \%$ compared to that of the PED + $\mathrm{N}_{3} @ P G D A 7$ group, with $40 \%$ animal survived in 100-days post treatment (Fig. 6H). TUNEL staining of the tumor sections revealed obvious apoptosis of the tumor cells in the PED + $\mathrm{N}_{3} @ P G D A 7+$ PDT group, suggesting combination of PDT and BRD4 degradation with the bioorthogonal NPs cumulatively induced apoptosis of the tumor cells in vivo (Fig. 6J). Semi-quantitative analysis of the TUNEL staining data further revealed that PED + N $3 @$ PGDA7 + Laser 15.2- and 4.2-fold more efficiently induced apoptosis of the tumor cells than ARV771 and PED + $\mathrm{N}_{3} @ P G D A 7$, respectively (Supplementary Fig. 27). Treatment-induced apoptosis of the tumor cells was further confirmed by H\&E staining of the tumor sections ex-vivo (Fig. 6K). Moreover, negligible body weight loss and histopathological damage of the major organs were observed during the experimental period, verifying satisfying biosafety of the POLY-PROTAC NPs (Fig. 61 and Supplementary Fig. 43).

IHC examination of the tumor tissue demonstrated significantly decreased BRD4 expression in vivo (Fig. 6L). Semi-quantitative analysis of the IHC images further determined that PED + $\mathrm{N}_{3}$ @PGDA7 2.6-fold more efficiently downregulated BRD4 expression than $\mathrm{N}_{3} @ P G D A 7$ NP alone, validating increased intratumoral ARV771 distribution via the in-situ bioorthogonal reaction contributed to BRD4 degradation in vivo (Fig. 6M). Noticeably, the combination of PDT with PED + $\mathrm{N}_{3} @ P G D A 7$ further promoted BRD4 degradation compared to PED + $\mathrm{N}_{3} @ P G D A 7$ due to PDT-enhanced intracellular release of the ARV771 PROTAC in vivo (Figs.6M, N). Noticeably, combinatory therapy by PED + $\mathrm{N}_{3} @ P G D A 7$ + Laser 1.8- and 3.0- 
fold more efficiently activated caspase-3 and degraded BRD4 than PED + $\mathrm{N}_{3} @ P G D 7$, verifying synergistic apoptosis-induction profile between PDT and BRD4 degradation (Fig. 60).

\section{Conclusion}

The heterobifunctional PROTACs with protein degradation ability has been recently investigated for cancer therapy ${ }^{4}$. However, clinical translation of the small molecular PROTACs severely suffers from their insufficient bioavailability and low tumor specificity. It remains one priority to develop novel PROTACs for tumor-specific protein degradation and minimizing the on-target but off-tumor adverse effects. A tumormicroenvironment-activatable POLY-PROTAC nanoplatform was thus engineered herein for tumor-specific delivery and potentiating antitumor performance of PROTACs.

In comparison with small molecular PROTAC counterparts, the POLY-PROTAC NPs possessed several distinct advantages for tumor-specific protein degradation and enhanced antitumor potency. First, the POLY-PROTAC NPs with MMP-2-liable PEG corona elongated blood circulation of the small molecular PROTACs, while the PEG corona was cleaved via intratumoral MMP-2 for facilitating tumor-specific ambulation and retention of the PROTACs. Second, the POLY-PROTAC NPs can be disintegrated in the intracellular acidic microenvironment. The PROTAC prodrug can thus be restored in the cytosol via GSHmediated cleavage of the disulfide bond. Thirdly, the PROTAC encapsulation capacity of the POLYPROTAC NPs can be readily tuned by adjusting the polymerization degree of PROTACs. Furthermore, we demonstrated that the POLY-PROTAC NPs can be adapted to bioorthogonal click reaction-enforced tumorspecificity of the POLY-PROTACs. Remarkably, the azide-modified POLY-PROTAC NPs showed 3.9-fold higher tumor accumulation than the small molecular counterpart via in-situ click reaction with the pretargeted NPs, and therefore further boost PROTAC-based cancer therapy. Last but not least, other kind of stimuli-activatable chemical bonds (e.g., thioketone, selenic or boric acid bond) ${ }^{35,36}$ can be utilized to achieve spatial-temporally PROTAC activation and reinforce the therapeutic performance.

In summary, a POLY-PROTAC prodrug strategy was developed for tumor-specific delivery of PROTAC in this study. We demonstrated for the first time that the extracellular MMP-2, intracellular acidity and reduction multiple stimuli-activatable POLY-PROTAC nanoplatform can achieve increased tumor accumulation, deep tumor penetration and enhanced protein degradation performance over the small molecular counterpart. The POLY-PROTAC nanoplatform with extracellular tumor acidity-triggered bioorthogonal click reaction remarkably enhanced tumor-specific PROTAC delivery. The clickable POLYPROTAC nanoplatform can be further enforced with PDT for protein degradation and combinatory therapy, which completely eradicated the MDA-MB-231 TNBC tumor. Extracellular acidic and MMP-2 microenvironment has been well-documented in various solid tumors. Therefore, the acidity-activatable bioorthogonal POLY-PROTAC nanoplatform can be further extended for combinatory therapy of a broad spectrum of cancer by integrating multiple therapeutic regimens (e.g., chemotherapeutics and immunotherapeutics). Taken together, the ingenious POLY-PROTAC nanoplatform might provide a novel avenue for potentiating PROTAC-based cancer therapy. 


\section{Author Statement}

J. Gao, H. Xu and H. Yu conceived the project and designed the study. Q. Zhu and X. Jiang synthesized the small molecule PROTACs and their methacrylate derivatives. J. Gao and B. Hou conducted the in-situ click reaction study. J. Gao synthesized the POLY-PROTACs and evaluated the protein degradation profiles in vitro and in vivo. J. Gao, L. Yang, Z. Zou and T. Wang conducted the anti-tumor study. J. Gao and $\mathrm{H}$. Yu analyze the data and wrote the initial manuscript. T. Xu, Z. Xu and H. Xu revised the manuscript.

\section{Declarations}

\section{Author statement}

J. Gao, H. Xu and H. Yu conceived the project and designed the study. Q. Zhu and X. Jiang synthesized the small molecule PROTACs and their methacrylate derivatives. J. Gao and B. Hou conducted the in situ bioorthogonal click reaction study. J. Gao synthesized the POLYTACs and evaluated the protein degradation profiles in vitro and in vivo. J. Gao, L. Yang and Z. Zou conducted the anti-tumor study. J. Gao and $\mathrm{H}$. Yu analyze the data and wrote the initial manuscript. Z. Xu, T. Xu and H. Xu revised the manuscript.

\section{Supporting Information}

Supporting Information is available from the author.

\section{Acknowledgements}

This work was supported by the National Natural Science Foundation of China $(51873228,22074043)$, International Cooperation Project of Science and Technology Commission of Shanghai Municipality (20430711800), the Youth Innovation Promotion Association of CAS (2014218), and Open Funds of State Key Laboratory of Drug Research, Shanghai Institute of Materia Medica, CAS (SIMM2105KF-12). The Mass Spectrometry System and the cell sorter BD Influx of the National Facility for Protein Science in Shanghai (NFPS), Shanghai Advanced Research Institute, CAS are gratefully acknowledged. All animal procedures were carried out under the guidelines approved by the Institutional Animal Care and Use Committee (IACUC) of the Shanghai Institute of Materia Medica, CAS.

\section{Conflict of Interest}

The authors declare no conflict of interest

\section{References}

1. Lai, A. C. \& Crews, C. M. Induced Protein Degradation: an Emerging Drug Discovery Paradigm. Nat. Rev. Drug Discov. 16, 101-114 (2017). 
2. Dale, B. et al. Advancing Targeted Protein Degradation for Cancer Therapy. Nat. Rev. Cancer 21, 638654 (2021).

3. Chamberlain, P. P. \& Hamann, L. G. Development of Targeted Protein Degradation Therapeutics. Nat. Chem. Biol. 15, 937-944 (2019).

4. Pettersson, M. \& Crews, C. M. PROteolysis TArgeting Chimeras (PROTACs)-Past, Present and Future. Drug Discov. Today 31, 15-27 (2019).

5. Skaar, J. R., Pagan, J. K. \& Pagano, M. SCF Ubiquitin Ligase-Targeted Therapies. Nat. Rev. Drug Discov. 13, 889-903 (2014).

6. Sakamoto, K. M. et al. Protacs: Chimeric Molecules that Target Proteins to the Skp1-Cullin-F Box Complex for Ubiquitination and Degradation. Proc. Natl Acad. Sci. USA 98, 8554 (2001).

7. Burslem, G. M. \& Crews, C. M. Proteolysis-Targeting Chimeras as Therapeutics and Tools for Biological Discovery. Cell 181, 102-114 (2020).

8. Backus, K. M. et al. Proteome-Wide Covalent Ligand Discovery in Native Biological Systems. Nature 534, 570-574 (2016).

9. Popovic, D., Vucic, D. \& Dikic, I. Ubiquitination in Disease Pathogenesis and Treatment. Nat. Med. 20, 1242-1253 (2014).

10. Schapira, M., Calabrese, M. F., Bullock, A. N. \& Crews, C. M. Targeted protein degradation: expanding the toolbox. Nat. Rev. Drug Discov. 18, 949-963 (2019).

11. Neklesa, T. K., Winkler, J. D. \& Crews, C. M. Targeted Protein Degradation by PROTACs. Pharmacol Ther 174, 138-144 (2017).

12. Bushweller, J. H. Targeting Transcription Factors in Cancer-from Undruggable to Reality. Nat. Rev. Cancer 19, 611-624 (2019).

13. Raina, K. et al. PROTAC-induced BET Protein Degradation as a Therapy for Castration-Resistant Prostate Cancer. Proc. Natl Acad. Sci. USA 113, 7124-7129 (2016).

14. Lu, J. et al. BRD4 Degradation By Protacs Represents a More Effective Therapeutic Strategy Than BRD4 Inhibitors in DLBCL. Blood 126, 2050-2050, (2015).

15. Moreau, K. et al. Proteolysis-Targeting Chimeras in Drug Development: A safety perspective. $\mathrm{Br} J$ Pharmacol 177, 1709-1718 (2020).

16. Reynders, M. et al. PHOTACs Enable Optical Control of Protein Degradation. Sci. Adv. 6, eaay5064 (2020). 
17. Cotton, A. D., Nguyen, D. P., Gramespacher, J. A., Seiple, I. B. \& Wells, J. A. Development of AntibodyBased PROTACs for the Degradation of the Cell-Surface Immune Checkpoint Protein PD-L1. J Am Chem Soc 143, 593-598 (2021).

18. Pillow, T. H. et al. Antibody Conjugation of a Chimeric BET Degrader Enables in vivo Activity. ChemMedChem 15, 17-25 (2020).

19. Banik, S. M. Nature 584 (2020).

20. Maneiro, M. A. et al. Antibody-PROTAC Conjugates Enable HER2-Dependent Targeted Protein Degradation of BRD4. ACS Chem. Biol. 15, 1306-1312 (2020).

21. Liu, J. et al. Cancer Selective Target Degradation by Folate-Caged PROTACs. J Am Chem Soc 143, 7380-7387 (2021).

22. He, S. et al. Aptamer-PROTAC Conjugates (APCs) for Tumor-Specific Targeting in Breast Cancer. Angew. Chem. Int. Ed., (2021).

23. Xue, G., Wang, K., Zhou, D., Zhong, H. \& Pan, Z. Light-Induced Protein Degradation with Photocaged PROTACs. J Am Chem Soc 141, 18370-18374 (2019).

24. Liu, J. et al. Light-induced Control of Protein Destruction by Opto-PROTAC. Sci. Adv. 6, eaay5154 (2020).

25. Hou, B. et al. Engineering Stimuli-Activatable Boolean Logic Prodrug Nanoparticles for Combination Cancer Immunotherapy. Adv. Mater 32, e1907210 (2020).

26. Zhou, F. et al. Engineering Chameleon Prodrug Nanovesicles to Increase Antigen Presentation and Inhibit PD-L1 Expression for Circumventing Immune Resistance of Cancer. Adv. Mater 33, 2102668 (2021).

27. Wang, Y. et al. A Nanoparticle-Based Strategy for the Imaging of a Broad Range of Tumours by Nonlinear Amplification of Microenvironment Signals. Nat Mater 13, 204-212 (2014).

28. Yang, B., Gao, J., Pei, Q., Xu, H. \& Yu, H. Engineering Prodrug Nanomedicine for Cancerlmmunotherapy. Adv Sci 7, 2002365 (2020).

29. Riley, R. S., June, C. H., Langer, R. \& Mitchell, M. J. Delivery Technologies for Cancer Immunotherapy. Nat. Rev. Drug Discov. 18, 175-196 (2019).

30. Ma, X. et al. Ultra-pH-Sensitive Nanoprobe Library with Broad pH Tunability and Fluorescence Emissions. J Am Chem Soc 136, 11085-11092 (2014).

31. Wang, Y. et al. Disulfide Bond Bridge Insertion Turns Hydrophobic Anticancer Prodrugs into Selfassembled Nanomedicines. Nano Lett 14, 5577-5583 (2014). 
32. Feng, B. et al. Binary Cooperative Prodrug Nanoparticles Improve Immunotherapy by Synergistically Modulating Immune Tumor Microenvironment. Adv. Mater 30, 1803001 (2018).

33. Wang, W. et al. In situ Activation of Therapeutics Through Bioorthogonal Catalysis. Adv Drug Deliv Rev 176, 113893 (2021).

34. Qin, H. et al. Development of a Cancer Vaccine Using In Vivo Click-Chemistry-Mediated Active Lymph Node Accumulation for Improved Immunotherapy. Adv. Mater 33, 2006007 (2021).

35. Wang, H. et al. Metabolic Labeling and Targeted Modulation of Dendritic Cells. Nat Mater 19, 1244$1252(2020)$.

36. Bai, L. et al. Targeted Degradation of BET Proteins in Triple-Negative Breast Cancer. Cancer Res 77, 2476-2487 (2017).

37. Donati, B., Lorenzini, E. \& Ciarrocchi, A. BRD4 and Cancer: Going Beyond Transcriptional Regulation. Molecular Cancer 17, 164 (2018).

38. Cochran, A. G., Conery, A. R. \& Sims, R. J., 3rd. Bromodomains: a New Target Class for Drug development. Nat. Rev. Drug Discov. 18, 609-628 (2019).

39. Wang, D. et al. Acid-Activatable Versatile Micelleplexes for PD-L1 Blockade-Enhanced Cancer Photodynamic Immunotherapy. Nano Lett 16, 5503-5513 (2016).

40. Wang, T. et al. Intracellularly Acid-Switchable Multifunctional Micelles for Combinational Photo/Chemotherapy of the Drug-Resistant Tumor. ACS Nano 10, 3496-3508 (2016).

41. Liu, J., Chen, Q., Feng, L. \& Liu, Z. Nanomedicine for Tumor Microenvironment Modulation and Cancer Treatment Enhancement. Nano Today 21, 55-73 (2018).

42. Zhou, F. et al. Tumor Microenvironment-Activatable Prodrug Vesicles for Nanoenabled Cancer Chemoimmunotherapy Combining Immunogenic Cell Death Induction and CD47 Blockade. Adv. Mater 31, 1805888 (2019).

43. Piya, S. et al. BRD4 Proteolysis Targeting Chimera (PROTAC) ARV-825, Causes Sustained Degradation of BRD4 and Modulation of Chemokine Receptors, Cell Adhesion and Metabolic Targets in Leukemia Resulting in Profound Anti-Leukemic Effects. Blood 128, 748-748 (2016).

44. Song, R. et al. Acidity-Activatable Dynamic Nanoparticles Boosting Ferroptotic Cell Death for Immunotherapy of Cancer. Adv. Mater 33, 2101155 (2021).

45. Feng, B. et al. Self-Amplified Drug Delivery with Light-Inducible Nanocargoes to Enhance Cancer Immunotherapy. Adv. Mater 31, 1902960 (2019). 


\section{Figures}

a

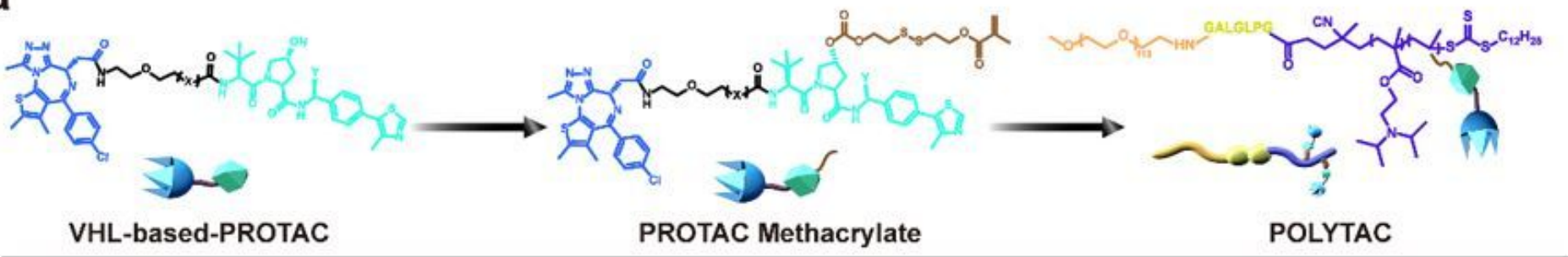

b

Pretargeted nanoparticle
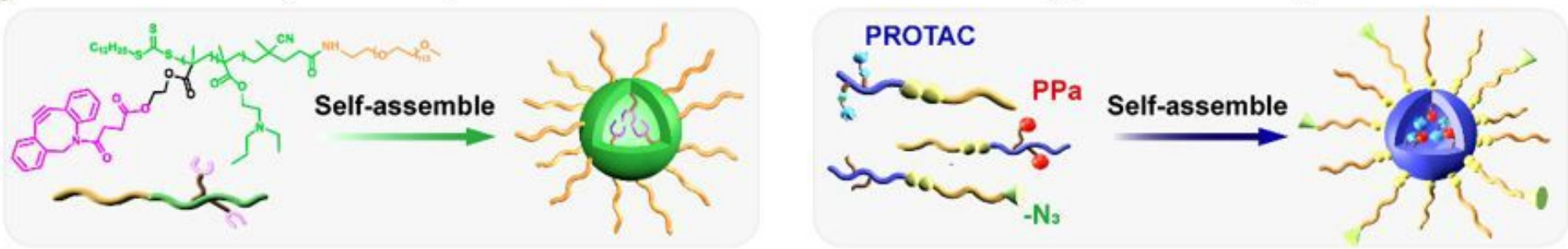

C First (i.v.)

Second (i.v.)
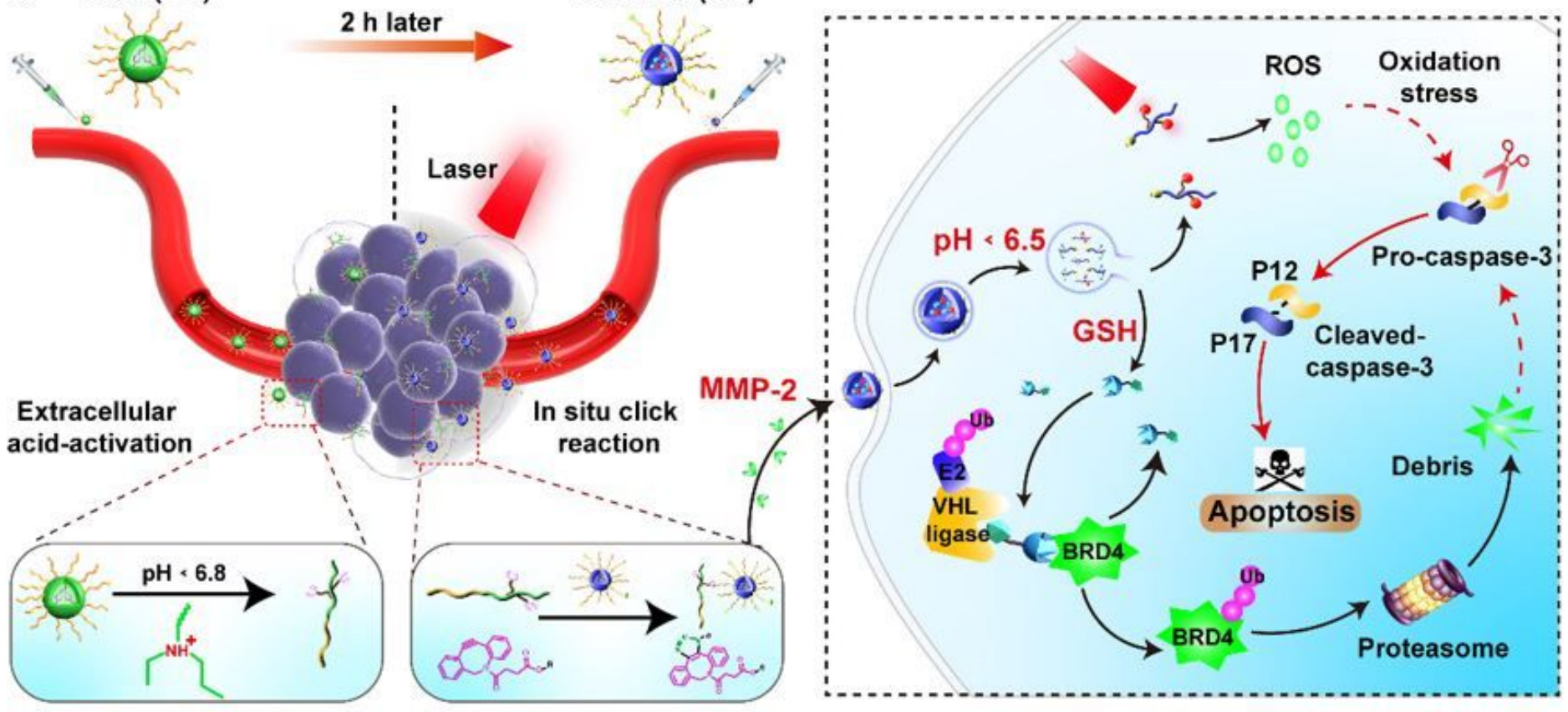

Figure 1

Schematic illustration of tumor microenvironment-activatable POLYTAC nanoparticles for bioorthogonal click reaction-enhanced cancer therapy. a, Synthesis of POLYTAC via RAFT polymerization approach, the PROTAC molecule was modified with disulfide linker and thereafter copolymerized with DPA using a MMP-2-liable macromolecular chain transfer agent; $\mathbf{b}$, Self-assemble of the BDCO-conjugated pretargeted nanoparticles, and the azide-modified POLYTAC nanoparticles; $\mathbf{c}$, Schematic demonstration of bioorthogonal strategy-promoted tumor distribution and combinatory tumor therapy with the POLYTAC nanoparticles, which displayed enhanced tumor distribution and deep penetration through in-situ click reaction with the pretargeted nanoparticles and deshielding of the PEG corona in the extracellular matrix 
of tumor. The POLYTAC nanoparticles were then internalized with the tumor cells for BRD4 degradation and combinatory cancer therapy with PDT.

a

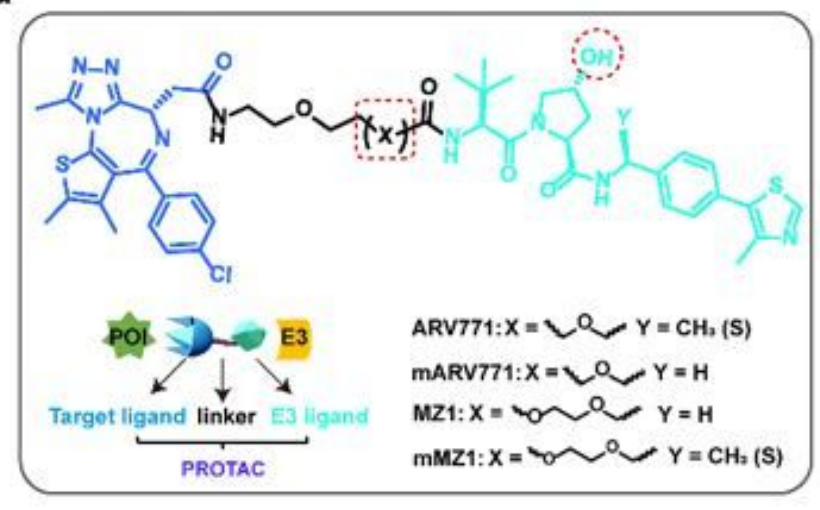

b
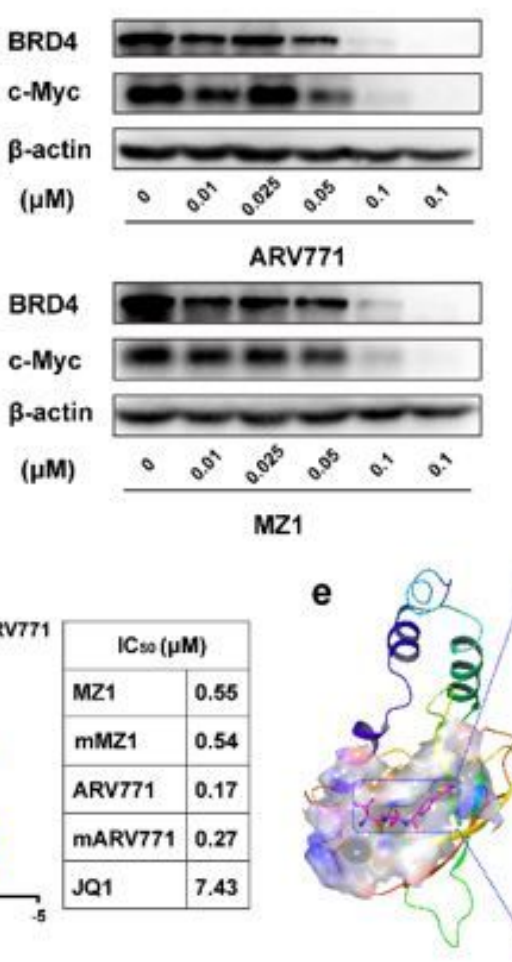

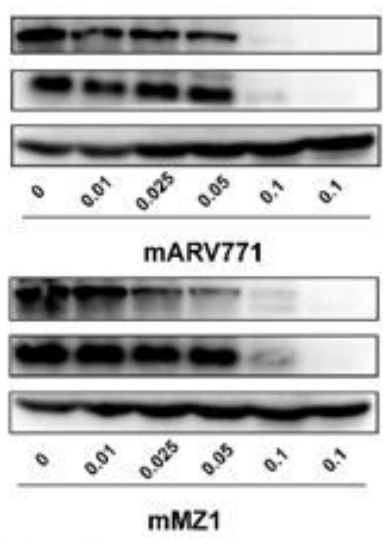

c

DCso

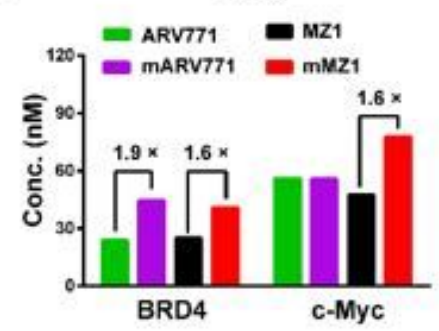

f

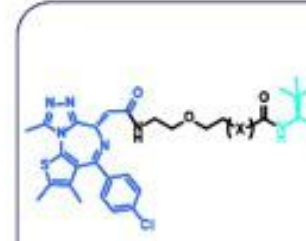

Me-ARV771: $z=-s^{3}$

Me-MZ1: $z=-$ s. $^{2}$

Me-O-ARV771: $z=$,
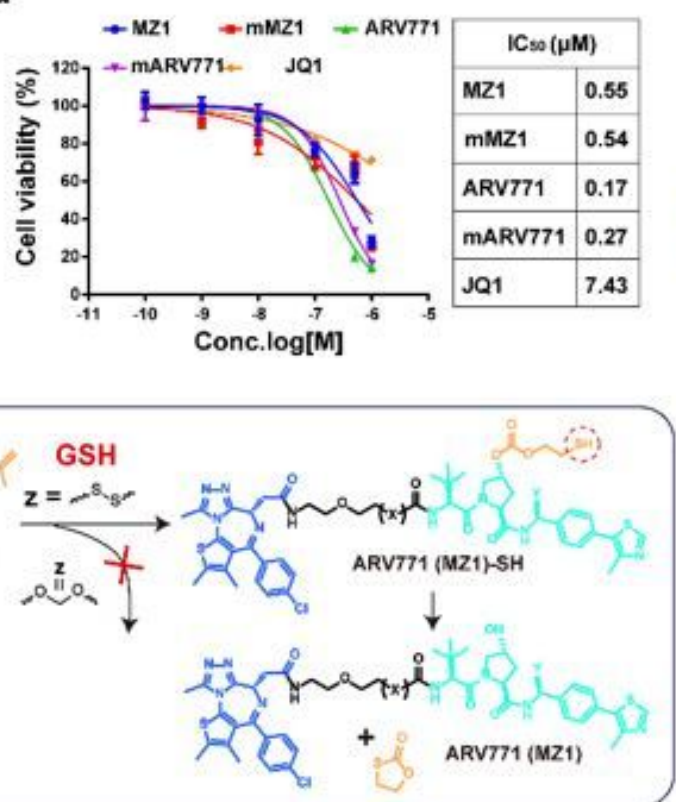

h
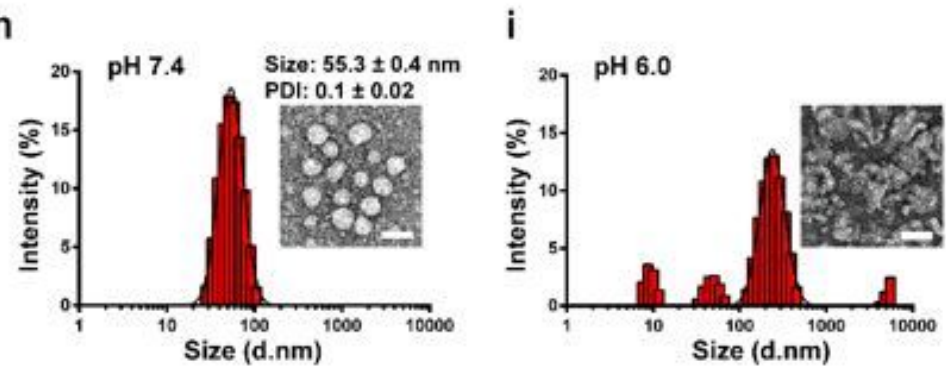

g

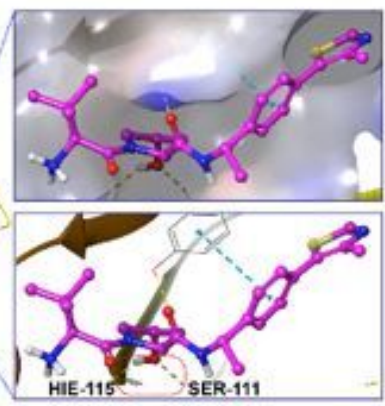

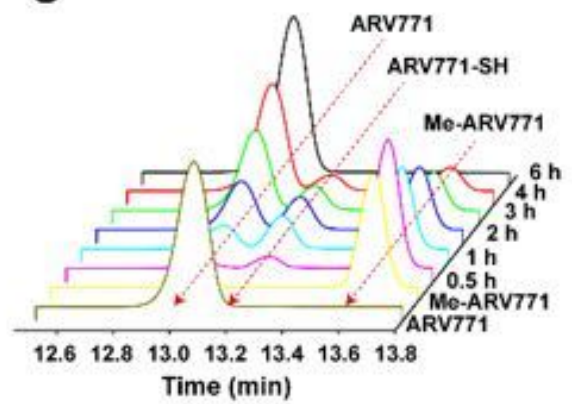
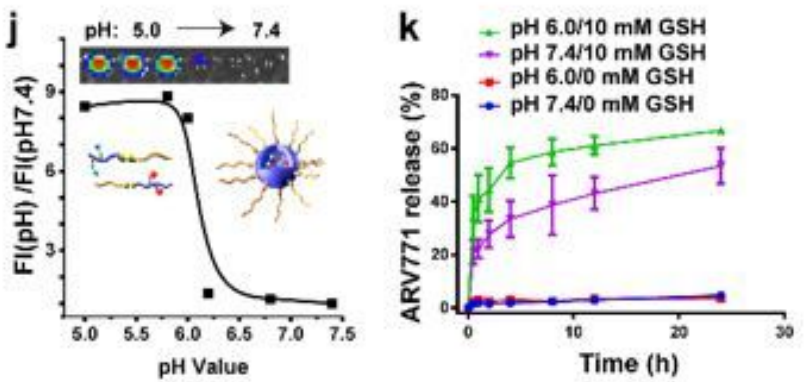

Figure 2

Synthesis and characterization of the PROTAC prodrug. $\mathbf{a}$, Synthesis of BRD4-targeted VHL PROTACs; $\mathbf{b}$, Western blot assay of PROTAC-mediated BRD4 degradation and c-Myc downregulation in the MDA-MB231 breast tumor cells after $24 \mathrm{~h}$ incubation; c, Western blot assay- determined half BRD4 degradation concentration $\left(\mathrm{DC}_{50}\right)$ of the PROTACS; $\mathbf{d}$, PROTACs efficiently inhibited proliferation of MDA-MB-231 cell 
in a dose-dependent manner in vitro (insert showed the $\mathrm{IC}_{50}$ of the indicated PROTACs and JQ1). The cells were incubated with the PROTACs or JQ1 for $72 \mathrm{~h}$ before subjected to CCK-8 assay; e, Molecular docking of VHL ligand (purple) binding with the VHL protein. The panorama (left) and detailed drawing (right) of dock; $\mathbf{f}$, Schematic illustration of GSH-mediated activation of ARV771 from the Me-ARV771; $\mathbf{g}$, HPLC plots of reduction-triggered ARV771 restoration via co-incubation with $5.0 \mathrm{mM}$ of DTT; $\mathbf{h}$, $\mathbf{i}$, Representative DLS data and TEM images of the PGD7 nanoparticle at (h) pH 7.4, or (i) pH 6.0; (j) Acidactivatable fluorescence profile of the PPa-labled PGDA7 nanoparticles (the fluorescence intensity was normalized with that determined at $\mathrm{pH} 7.4$ (insert showed the fluorescence image of the PGDA7 nanoparticle suspensions at different pHs); $\mathbf{k}$, GSH-triggered ARV771 release from the PGD7 nanoparticle at $\mathrm{pH} 7.4$ or $\mathrm{pH}$ of 6.0 (with or without $10 \mathrm{mM} \mathrm{GSH}$ addition). 
a

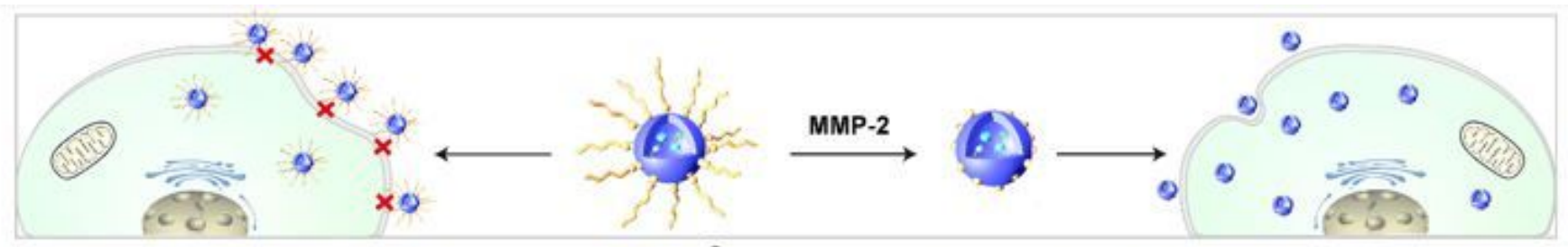

b
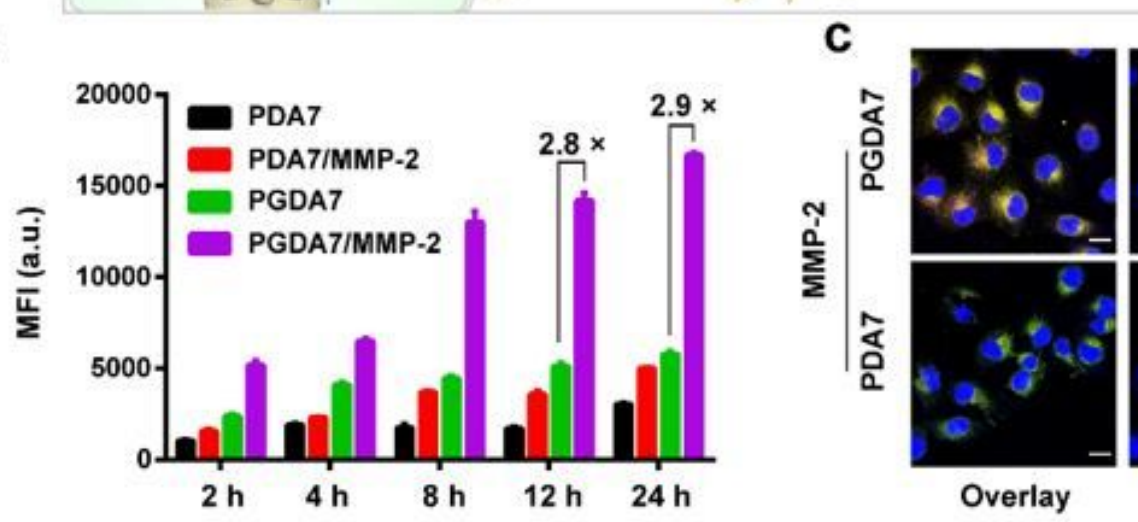

Overlay

e

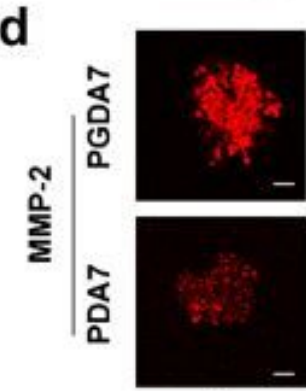

40

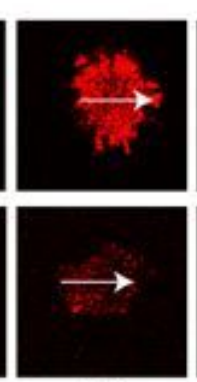

50

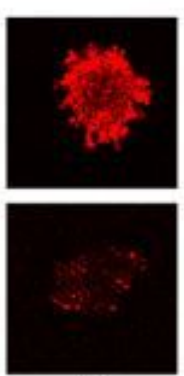

60

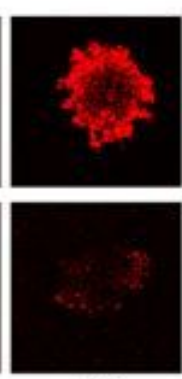

70

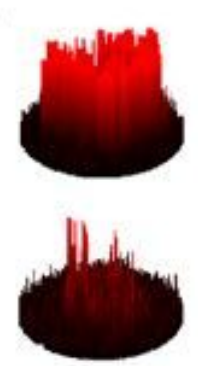

$50 \mu \mathrm{m}$

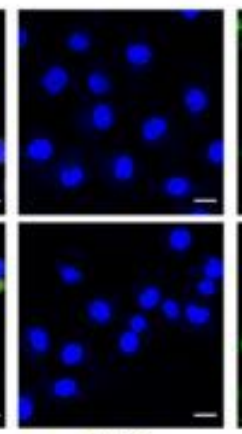

DAPI

f

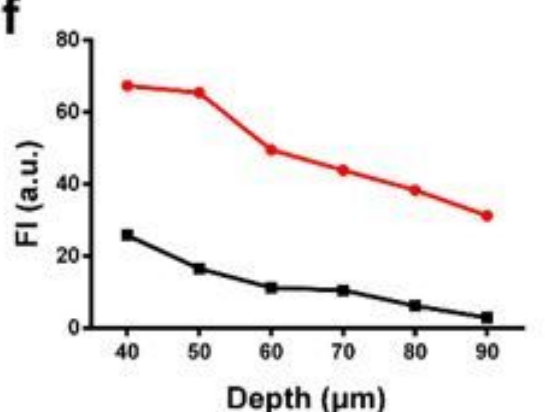

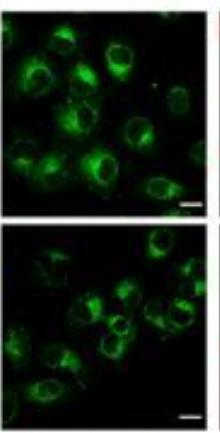

Lysotracker

$\mathrm{PPa}$
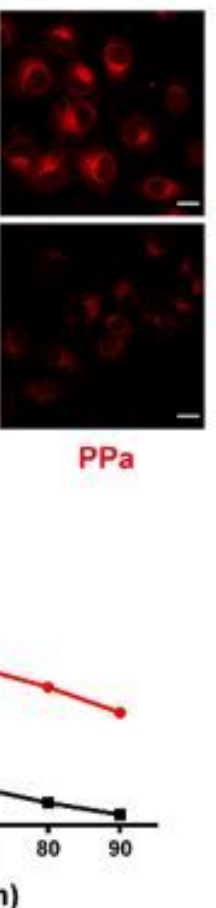

Section depth $(\mu \mathrm{m})$

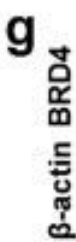

$(\mu \mathrm{M}) \frac{00^{0} 0^{\wedge} 0^{2} 0^{5}}{\text { PDM }}$

k

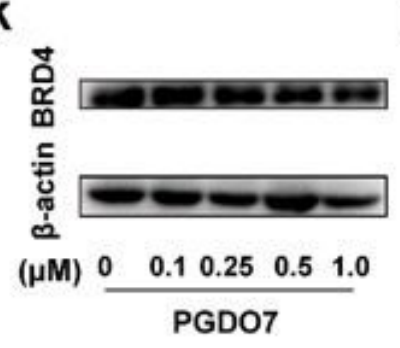

I h

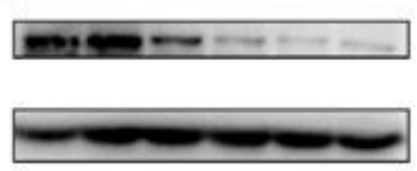

$\frac{00^{0} 0^{\circ} 0^{2} 0^{5}}{\text { PGDM }}$

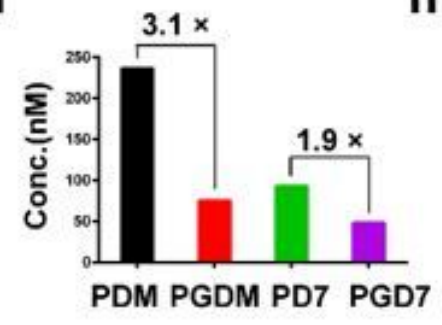

m

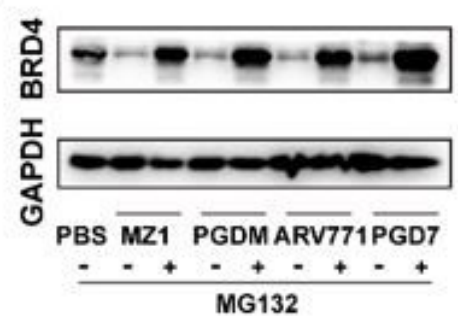

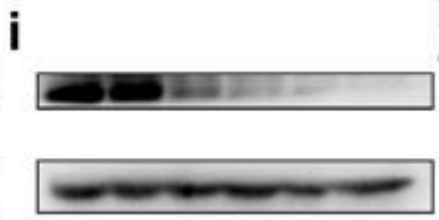

$\frac{00^{n} 0^{n} 0^{p} 0^{5}}{\text { PD7 }}$

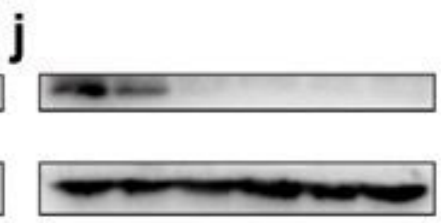

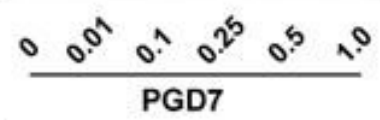

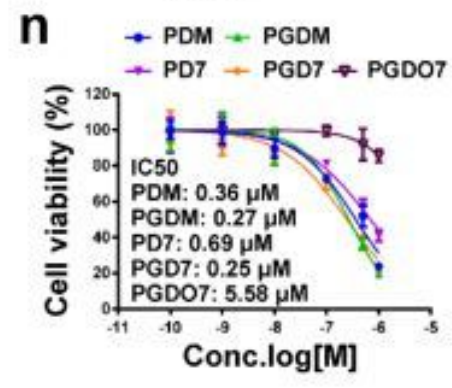

Figure 3

Cellular uptake, tumor penetration and BRD4 degradation performance of the sheddable POLYTAC nanoparticle in vitro. a, Cartoon illustration of MMP-2-triggered cleavage of the PEG corona and increased cellular uptake of the PGD7 nanoparticles in vitro; b, Flow cytometric analysis of cellular uptake of PGDA7 and PDA7 nanoparticles in vitro (The nanoparticles were pre-treated with $0.2 \mathrm{mg} / \mathrm{mL}$ of MMP-2 for $1 \mathrm{~h})$; c, CLSM examination of intracellular distribution of the POLYTAC nanoparticles upon $12 \mathrm{~h}$ 
incubation (scale bar $=20 \mu \mathrm{m}$ ); d-f, MMP-2-responsive PGDA7 nanoparticles displayed increased penetration ability in the MCSs of MDA-MB-231 cells in vitro than the MMP-2-non-resposive PDA7 counterpart. (d) CLSM examination of PGDA7 and PDA7 nanoparticle distribution post $12 \mathrm{~h}$ incubation in vitro; (e) 2.5-D reconstruction of the CLSM images at a depth of $50 \mu \mathrm{m}$; (f) Fluorescence intensity of the central region versus Z-axis depth; $\mathbf{g - j}$, The reduction-activatable POLYTAC nanoparticle efficiently degraded BRD4 protein in the MDA-MB-231 cells in vitro. (h-j) Western blot assay of BRD4 degradation in MDA-MB-231 cells with various GSH-sensitive POLYTAC nanoparticles in vitro upon $24 \mathrm{~h}$ incubation; $\mathbf{k}$, Western blot assay of BRD4 expression in the PGD07 nanoparticle-treated MDA-MB-231 cells in vitro; I, $\mathrm{DC}_{50}$ of the POLYTAC nanoparticles for degrading BRD4 in the MDA-MB-231 cells in vitro; m, POLYTAC nanoparticle degraded POI via the ubiquitin-proteasome system, western blot assay of BRD4 expression in the MDA-MB-231 cell with or without MG132 incubation (MZ1/ARV771 concentration of $1.0 \mu \mathrm{M}$ and MG132 concentration of $5.0 \mathrm{mM}$, respectively); $\mathbf{n}$, PGDM and PGD7 nanoparticles efficiently inhibited the proliferation of MDA-MB-231 cells in vitro (The POLYTAC nanoparticle were named according to the PROTAC and components integrated, P: PEG chain; G: MMP-2-liable GPLGLAG spacer; D: acid-activatable DPA groups; 7: disulfide bond-bearing ARV771 methacrylate; M: disulfide bond-bearing MZ1 methacrylate; 07: ethylene group-bearing ARV771 methacrylate; A: Pyropheophorbide a, PPa). 

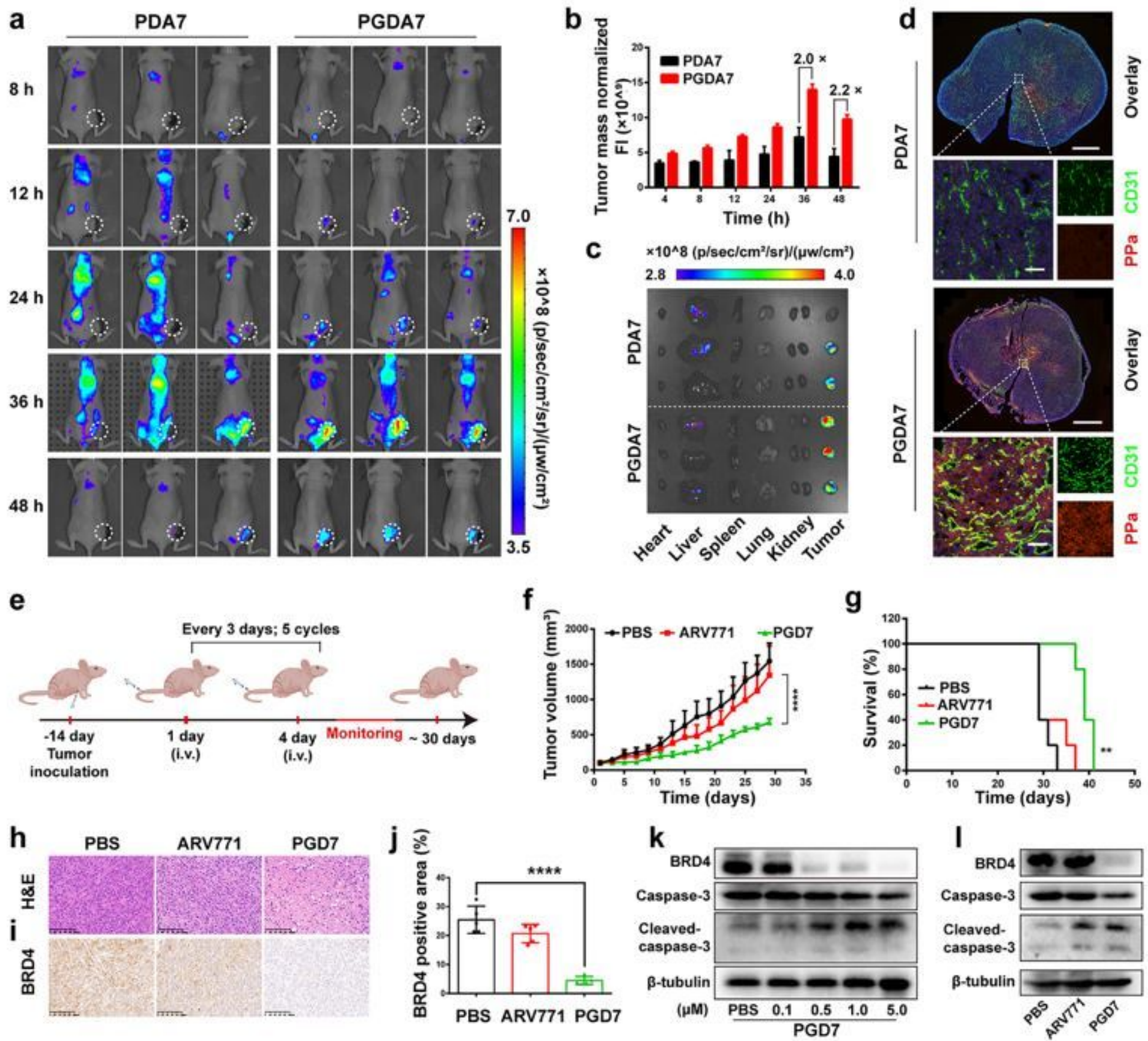

Figure 4

Biodistribution and anti-tumor performance of the POLYTAC nanoparticles in vivo. a, Fluorescence images of POLYTAC nanoparticle distribution in MDA-MB-231 tumor-bearing nude mice in vivo, the MMP2-activatible PGDA7 nanoparticle specifically accumulated at the tumor site in vivo; $\mathbf{b}$, Normalized fluorescence intensities of the tumor tissue, and (c) Fluorescence images of the major organs and the tumor tissue ex-vivo (the major organs and tumors were harvested $48 \mathrm{~h}$ post-injection); d, CLSM images of ex-vivo tumor sections at $48 \mathrm{~h}$ post-injection (top panel scale bar $=2.0 \mathrm{~mm}$, bottom panel scale bar $=$ $50 \mu \mathrm{m}) ; \mathbf{e}$, Treatment schedule of POLYTAC nanoparticle-performed antitumor study in MDA-MB-231 tumor-bearing nude mice; $\mathbf{f - g}$, PGD7 nanoparticle efficiently suppressed MDA-MB-231 tumor growth without obvious adverse effect. (f) Tumor growth curves, and (g) survival plots of the tumor-bearing 
Balb/c nude mice upon Poly-PROTAC nanoparticle treatments ( $n=5)$; $h$, H\&E staining of the tumor sections at the end of antitumor study (scale bar $=100 \mu \mathrm{m}$ ); i-I, The PGD7 nanoparticles degraded BRD4 protein and activated caspase-3 in the tumor cell in vitro and in vivo. (i) IHC staining of BRD4 expression in the tumor sections ex-vivo (scale bar $=100 \mu \mathrm{m}$ ), and (j) Semi-quantitative analysis of IHC-determined BRD4 expression; k\&l, Western blot assay of PGD7 POLYTAC-induced BRD4 degradation and caspase-3 activation in MDA-MB-231 tumor cells ( $\mathbf{k})$ in vitro, and (I) in vivo (the tumors were harvested at the second day post five-cycles treatments).
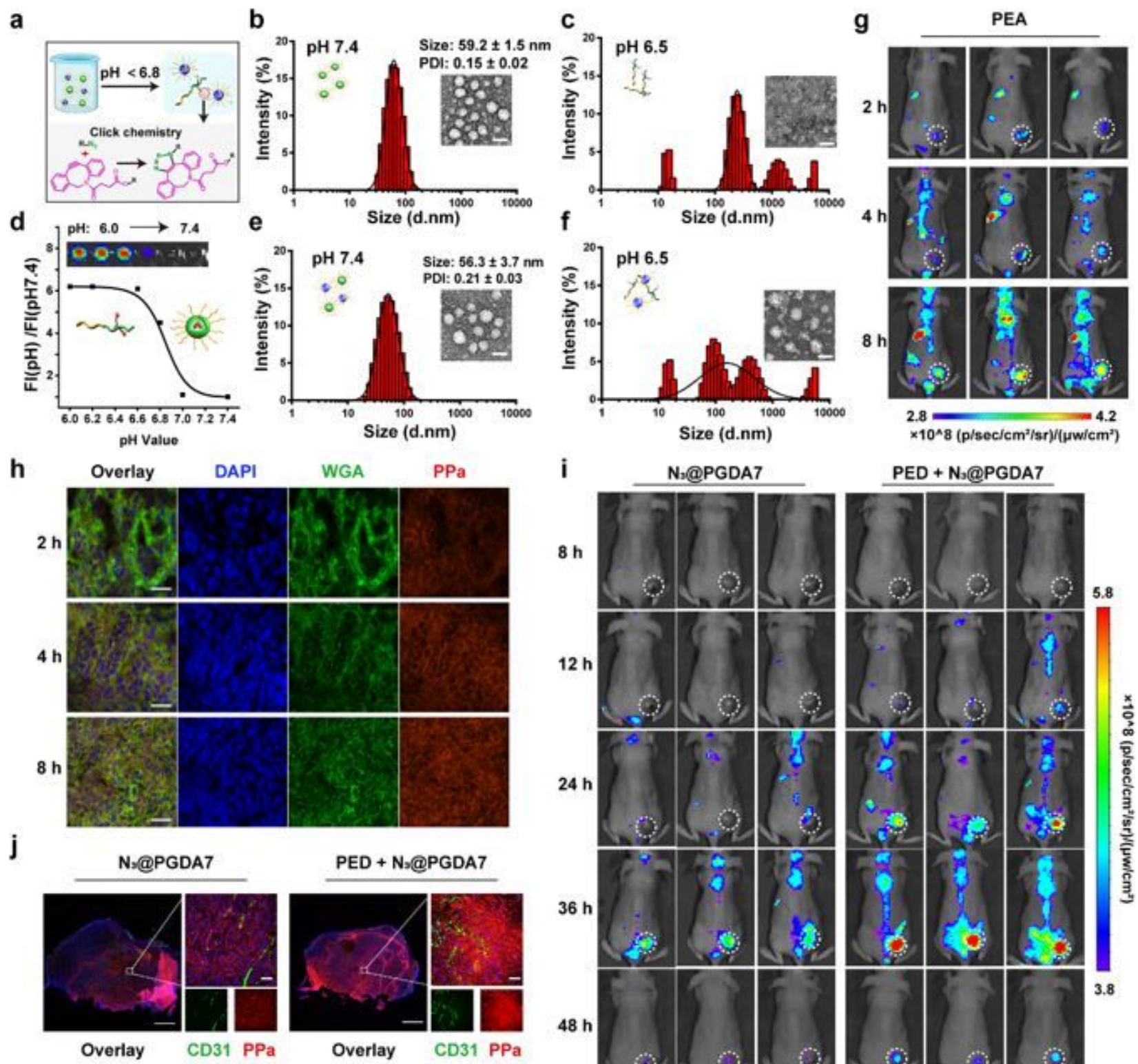

$\times 10^{\wedge} 8\left(\mathrm{p} / \mathrm{sec} / \mathrm{cm}^{2} / \mathrm{sr}\right) /\left(\mu \mathrm{w} / \mathrm{cm}^{2}\right)$
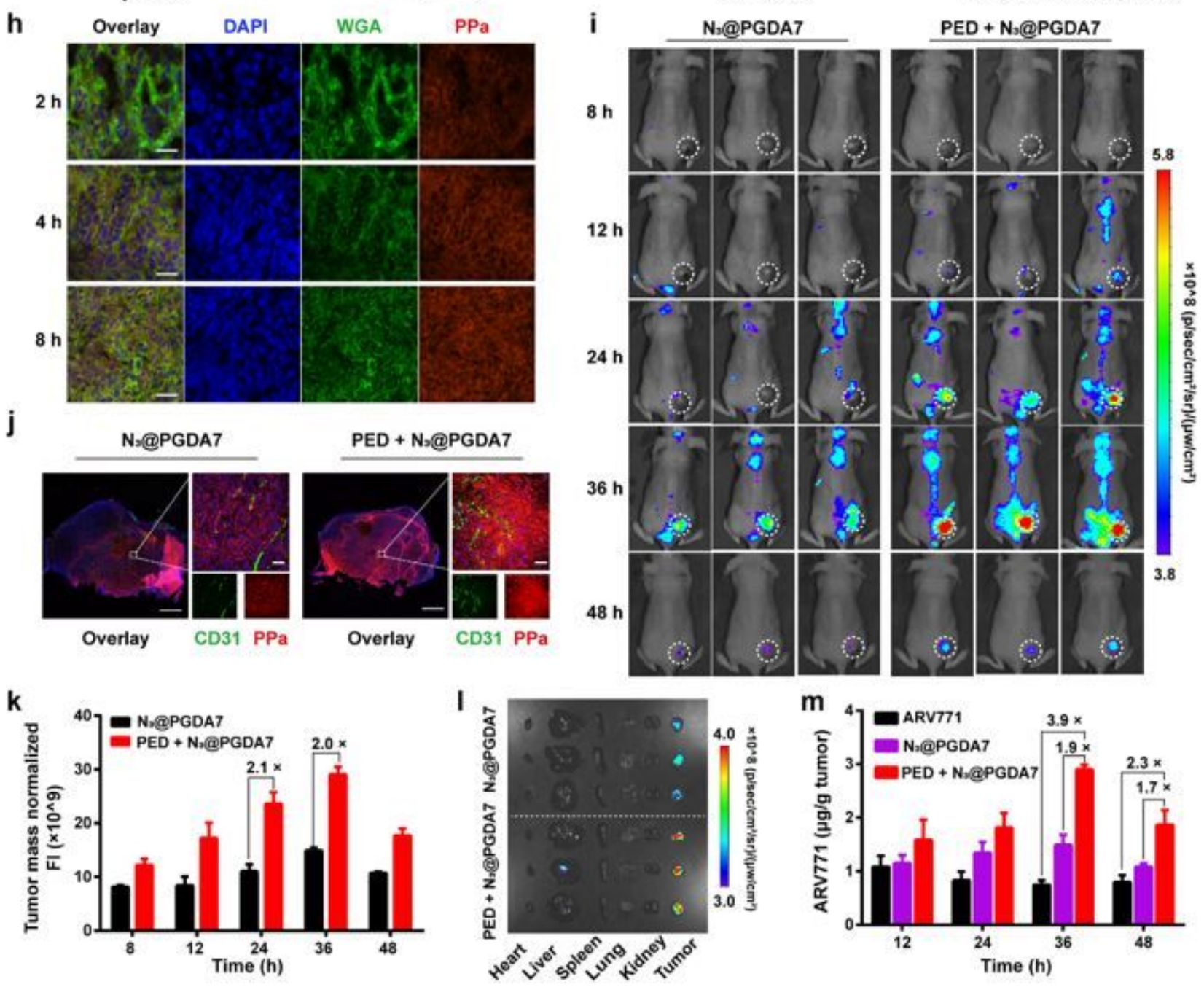


\section{Figure 5}

\section{Bioorthogonal click reaction improved tumor accumulation of the POLYTAC nanoparticle in vivo. a,}

Diagrammatic illustration of tumor-specific delivery of the POLYTAC nanoparticles via bioorthogonal click reaction with the extracellular tumor acidity-activatable pretargeted nanoparticles; $\mathbf{b}$-d, DLS and TEM examination of the pretargeted nanoparticles at (b) $\mathrm{pH} 7.4$, and (c) $\mathrm{pH}$ 6.5. The PED pretargeted nanoparticles dissociated at the extracellular tumor acidity for release DBCO in vitro; (d) Normalized fluorescence plots of the pretargeted nanoparticle versus $\mathrm{pH}$ values (insert: fluorescence image of the PEA nanoparticle at different $\mathrm{pH}$ conditions); $\mathbf{e}, \mathbf{f}, \mathrm{DLS}$ and TEM examination of the particle distribution and morphology of the mixture of PED + $\mathrm{N}_{3} @$ PGD7 nanoparticles at (e) pH 7.4, and (f) 6.5. The bioorthogonal reaction occurred between the PED pretargeted nanoparticles and the $\mathrm{N}_{3} @ P G D 7$ POLYTAC nanoparticles at $\mathrm{pH} 6.5 ; \mathbf{g}, \mathbf{h}$, The pretargeted nanoparticles specifically accumulated at the tumor site and were activated with the tumor acidic $\mathrm{pH} ;(\mathbf{g})$ Fluorescence imaging of biodistribution of the PED nanoparticle in the MDA-MB-231 tumor bearing nude mice in vivo, and (h) CLSM examination the tumor section ex-vivo (scale bar $=50 \mu \mathrm{m}$ ); i-m, The PED nanoparticles increased tumor distribution of the $\mathrm{N}_{3} @ P G D 7$ nanoparticles via bioorthogonal click reaction in vivo; (i) Fluorescence imaging of MDA-MB231 tumor-bearing Balb/c nude mice, and (j) CLSM images of the tumor sections at $48 \mathrm{~h}$ post-injection $($ scale bar $=50 \mu \mathrm{m})($ The mice were i.v. injected with the PED pretargeted nanoparticles at a DBCO dose of $1.0 \mathrm{mg} / \mathrm{kg}$, and subsequently i.v. injected with the $\mathrm{N}_{3} @ P G D A 7$ nanoparticles at an azide dose of 0.055 $\mathrm{mg} / \mathrm{kg} 2 \mathrm{~h}$ post PED injection); The in-situ bioorthogonal click reaction remarkably increased ARV771 distribution in the tumor, (k) Intratumoral fluorescence intensity of $N_{3} @ P G D A 7$ nanoparticle-injected mice, and (I) Fluorescence imaging of the major organs $48 \mathrm{~h}$ post-injection; $\mathrm{m}$ ) HPLC-determined intratumoral ARV771 distribution. 
a

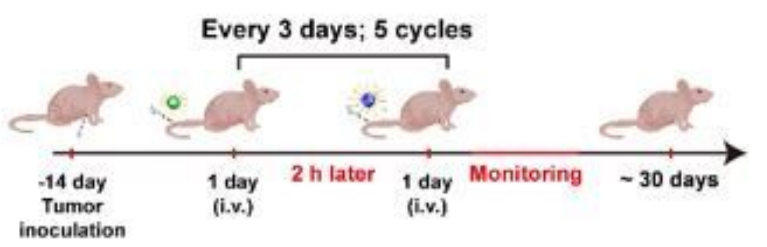

d

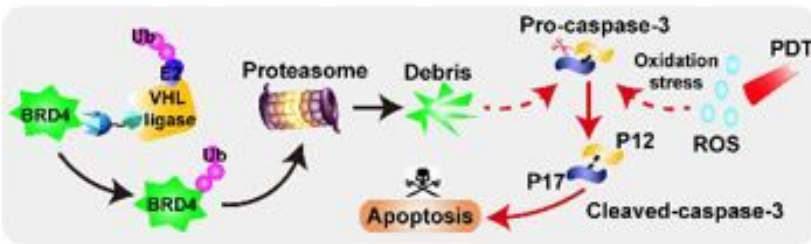

$\mathbf{f}$

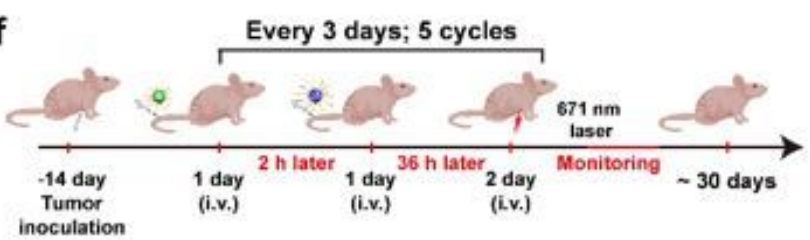

h

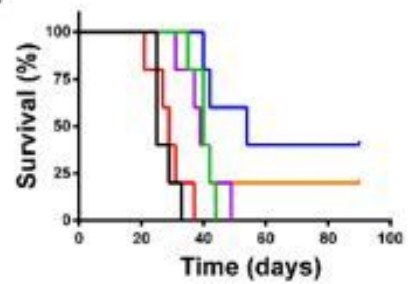

j

k
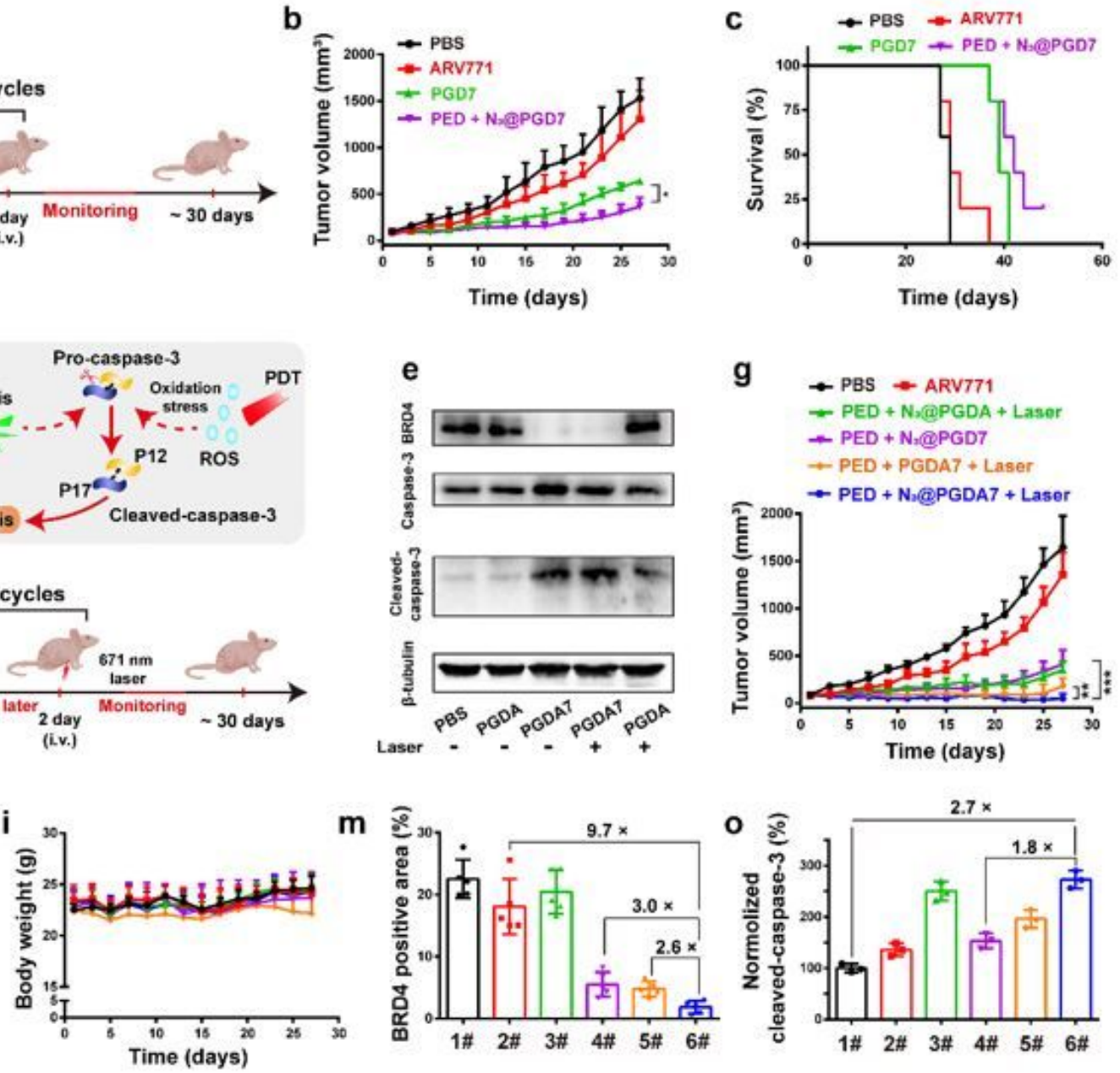

$-\mathrm{PED}+\mathrm{N}$ MPGD7

- $\mathrm{PED}+\mathrm{PGDA} 7$ + Laser

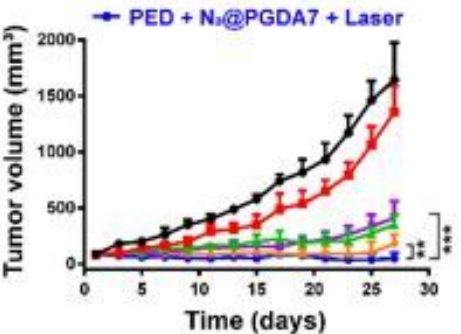

0

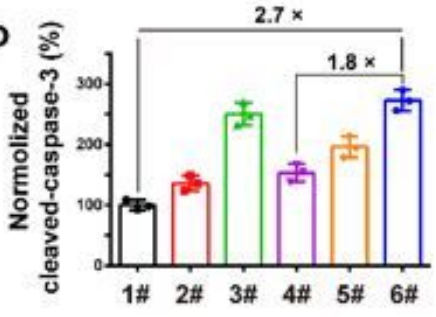

n

BRD4
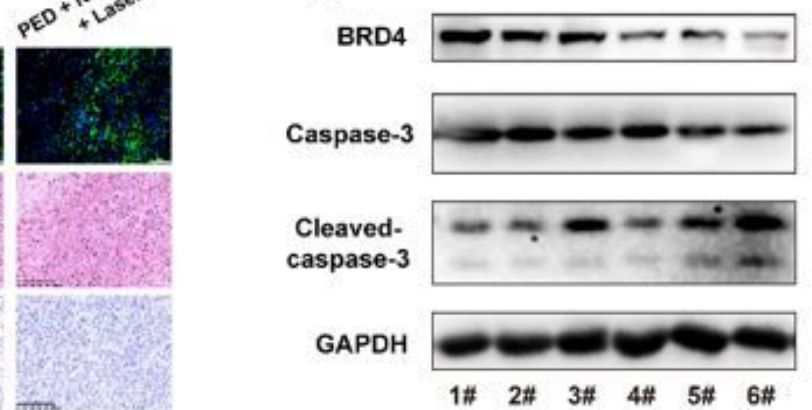

Figure 6

Anti-tumor performance of the bioorthogonal POLYTAC nanoparticle via BRD4 degradation and PDT. a, Experimental design for bioorthogonal POLYTAC nanoparticle-based anti-tumor study; $\mathbf{b}$, Averaged tumor growth curves after treating with various formulations (PBS, ARV771, PGD7 and PED + $N_{3} @ P G D 7, n=5$ ); c, Survival curves of the tumor-bearing mice; $d$, Schematic illustration of the bioorthogonal nanoparticlebased combinatory therapy of TNBC tumor via BRD4 degradation and caspase-3 activation in the tumor in vivo; $\mathbf{e}$, The combination of ARV771 and PDT cumulatively activated caspase- 3 of the tumor cells in vivo. Western blot assay of the tumor lysates upon different treatments; $\mathbf{f}$, Experimental schedule of bioorthogonal POLYTAC nanoparticle-performed combinatory therapy in MDA-MB-231 xenograft tumor 
model; g, Averaged tumor growth curves after treatment with various formulations (PBS, ARV771, PED + $\mathrm{N}_{3} @ P G D A$ + Laser, PED + N3 @PGD7, PED + PGD7 + Laser, PED + N N $_{3} @ P G D A 7$ + Laser, n = 5囚irradiation condition: $400 \mathrm{~mW} / \mathrm{cm}^{2}, 5.0 \mathrm{~min}$ ); $\mathbf{h}$, Survival curves, and $\mathbf{i}$, Body weight change of the tumor-bearing mice monitored during the experimental period; $\mathbf{j}$, TUNEL (blue: DAPI, green: apoptotic cell), and (k) H\&E staining of the tumor section 30-days post-treatment (scale bar $=100 \mu \mathrm{m}$ ); I, IHC analysis, and $\mathrm{m}$ ) Semiquantitated BRD4 expression in the tumor tissues; $\mathbf{n}$, Western blot assay, and (0) Normalized expression of the activated caspase-3 in the tumor lysates (1\#: PBS; 2\#: ARV771; 3\#: PED + N3@PGDA + Laser; 4\#:

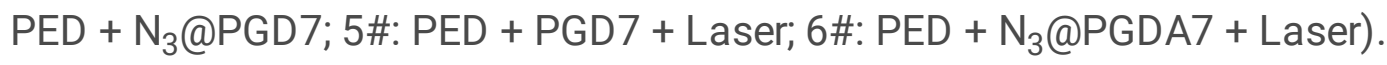

\section{Supplementary Files}

This is a list of supplementary files associated with this preprint. Click to download.

- SIGJ20211222Final.docx 